\title{
CpG islands' clustering uncovers early development genesin the human genome
}

\author{
R.O. Babenko ${ }^{1}$, A.V. Tsukanov ${ }^{1,2}$, A.G. Galieva1, Y.L. Orlov², V.N. Babenko ${ }^{2,3}$ \\ 1 Novosibirsk State University, Novosibirsk, Russia \\ 2 Institute of Cytology and Genetics SB RAS, Novosibirsk, Russia \\ *e-mail:rbab@yandex.ru
}

Key words: non-coding RNA, transcriptomics, stress response, crop plants

Motivation and Aim: We address the problem of the annotation of CpG islands (CGIs) clusters in the human genome. $\mathrm{CpG}$ dinucleotide rich genome regions, also known as $\mathrm{CpG}$ islands (CGIs), are important functional elements of vertebrate genomes [1]. In particular, in the majority of vertebrate genes, $\mathrm{CpG}$ islands coincide with gene promoter areas. In some cases, the transcription from $\mathrm{CpG}$-island containing promoters is bidirectional, this is related to self-complementarity of $\mathrm{CG}$ dinucleotides. CGIs are the key contributors to global methylation landscapes. Degenerate content of CGIs (biased CG frequency) assumes a higher probability of tandem repeats and palindromes inside a CGI.

Methods and Algorithms: We have used own program scripts for tandem repeats and CGI counts. We used a CGI clustering method that is robust relative to the tandem duplication search A set of CGIs was retrieved from the table cpgIslandExt (www.genome.ucsc. edu; version hg19). To identify significant CGI clustering, the human genome was split into $10 \mathrm{~Kb}$ non overlapping segments (bins) (243 785 bins in total). The number of CGIs per bin (CGI density) was assessed as a total number of CGIs divided by the number of bins. The expected number of CGIs per segment was approximated using a Poisson distribution

Results: Upon analyzing gene content within CGIs clusters, piRNA, tRNA, and miRNAencoding genes were found as well as $\mathrm{CpG}$-rich homeobox genes reported previously. Chromosome-wide CGI density is positively correlated with replication timing, confirming that CGIs may serve as open chromatin markers. Early embryonic stage expressed KRAB-ZNF genes abundant at chromosome 19 were found to be interlinked with CGI clusters.

Conclusion: We detected that a number of long CGIs and CGI clusters are, in fact, tandem copies with multiple annotated macrosatellites and paralogous genes. This finding implies that tandem expansion of CGIs may serve as a substrate for non-homologous recombination events [1].

Acknowledgements: The research has been supported by RFBR. Computing done at Siberian Supercomputer center SB RAS was supported by budget project 0324-20180017.

\section{References}

1. Babenko V.N., Bogomolov A.G., Babenko R.O., Galieva E.R., Orlov Y.L. (2018) CpG islands' clustering uncovers early development genes in the human genome. Computer Science Information Systems. 15(2):473-485. 